\title{
Hyperdense Posterior Cerebral Artery Sign in a Setting of Spontaneous Vertebral Artery Dissection: A Blessing in Disguise?
}

\author{
Sankalp Gokhale Carmelo Graffagnino \\ Duke University School of Medicine, Durham, N.C., USA
}

\section{Key Words}

Hyperdense posterior cerebral artery · Dissection

\begin{abstract}
Objective: To report a case of a hyperdense posterior cerebral artery (PCA) sign in the setting of spontaneous vertebral artery dissection. Clinical Presentation and Intervention: A 28-year-old, previously healthy female presented with rapidly progressive coma. A noncontrast computerized tomographic (CT) scan showed a hyperdense PCA sign, which prompted an urgent arteriogram. She was found to have spontaneous vertebral artery dissection with an occluding thrombus. She underwent intra-arterial thrombolysis with tissue plasminogen activator. Follow-up magnetic resonance imaging showed an area of acute infarction in the medial temporal and occipital regions corresponding to the area supplied by the left PCA. The patient was started on systemic anticoagulation therapy with intravenous heparin. She showed slow and continued recovery but was left with significant neurological deficits that required posthospital discharge to a long-term rehabilitation facility. Conclusion: This case showed that the hyperdense PCA sign on a noncontrast CT scan necessitated an emergent CT
\end{abstract}

angiogram that showed vertebral artery dissection and a devastating vascular occlusion. Hence, we suggest an early intervention that may allow for potential revascularization therapy.

C 2013 S. Karger AG, Basel

\section{Introduction}

The hyperdense middle cerebral artery (MCA) sign is a well-described radiological sign on noncontrast computerized tomographic (CT) scans in cases of acute ischemic stroke [1-3]. It suggests acute thromboembolic occlusion of the MCA. However, a hyperdense posterior cerebral artery (PCA) sign is not commonly encountered [4]. Further, presence of this sign in the setting of spontaneous vertebral artery dissection has not been described. Presence of a hyperdense sign is usually associated with a larger area of infarction and poor outcome, therefore early recognition and intervention are critical. We herein present a rare case of the hyperdense PCA sign in the setting of spontaneous vertebral artery dissection.

\begin{tabular}{ll}
\hline KARGER & $\begin{array}{l}\text { ○ 2013 S. Karger AG, Basel } \\
1011-7571 / 14 / 0231-0086 \$ 39.50 / 0 \quad \text { Karger }\end{array}$ \\
E-Mail karger@karger.com & $\begin{array}{l}\text { This is an Open Access article licensed under the terms of the } \\
\text { Creative Commons Attribution-NonCommercial 3.0 Un- } \\
\text { ported license (CC BY-NC) (www.karger.com/OA-license), } \\
\text { applicable to the online version of the article only. Distribu- } \\
\text { tion permitted for non-commercial purposes only. }\end{array}$
\end{tabular}




\section{Case Report}

A 28-year-old, previously healthy female presented to the emergency department with acute onset of headache and vomiting. She noted an unusual progressive throbbing headache at the back part of her neck just before going to sleep. The headache was worse after waking up in the morning. She was somnolent, complained of nausea with several episodes of emesis leading to a prompt evaluation in the emergency department. Her blood pressure was $130 / 60 \mathrm{~mm}$ $\mathrm{Hg}$, heart rate 106 beats/min and respiratory rate 26-30 breaths/ min. She was drowsy with small sluggishly reactive pupils. Formal neurological examination was difficult due to her drowsiness. Her condition rapidly deteriorated, progressing to coma and requiring endotracheal intubation for airway protection.

There was no significant medical or surgical history. She was on birth control pills but was not on any other outpatient medications. There was no family history of hypercoagulable state or premature strokes. There was no history of recent neck trauma or chiropractor visit.

An urgent noncontrast CT scan of the head showed no evidence of acute hemorrhage. However, there was evidence of a 'hyperdense left PCA' (fig. 1a), suggestive of a thromboembolic process in the left PCA. She was not a candidate for intravenous thrombolysis due to unclear onset of symptoms at least several hours before presentation. Decision was made to proceed with an urgent 4-vessel digital subtraction arteriogram to evaluate intracranial as well as extracranial circulation. The arteriogram showed evidence of left vertebral artery dissection, an occlusive thrombus in the left vertebral artery and embolic occlusion of the left PCA and basilar artery. This was thought to be spontaneous dissection in view of the negative history of neck trauma or manipulation in the recent past. She underwent intra-arterial thrombolysis with tissue plasminogen activator. She was then transferred to the neurointensive care unit. Follow-up magnetic resonance imaging was performed after intra-arterial thrombolysis (fig. 1b) which showed an area of acute infarction in the medial temporal and occipital region corresponding to the area supplied by the left PCA. In addition there was evidence of acute infarction in bilateral pontomedullary regions, corresponding to the basilar artery occlusion secondary to vertebral artery dissection. She was started on systemic anticoagulation therapy with intravenous heparin (with transition to oral warfarin) $24 \mathrm{~h}$ after undergoing intra-arterial thrombolysis. Her blood pressure was allowed to autoregulate with a goal systolic blood pressure of $140-180 \mathrm{~mm} \mathrm{Hg}$. Intermittent vasopressors were used to achieve this goal. This was done to maximize cerebral perfusion especially in the posterior circulation in the acute stage (first 48-72 h, after recanalization therapy). General intensive care support was provided. She had a prolonged hospital course in the neurointensive care unit including tracheostomy and gastrostomy feeding tube placement. She showed slow and continued recovery but was left with significant neurological deficits requiring posthospital discharge to a long-term rehabilitation facility.

\section{Discussion}

The hyperdense PCA sign in this case was due to spontaneous vertebral dissection leading to thrombus formation in the vertebral artery lumen. However, in other re-

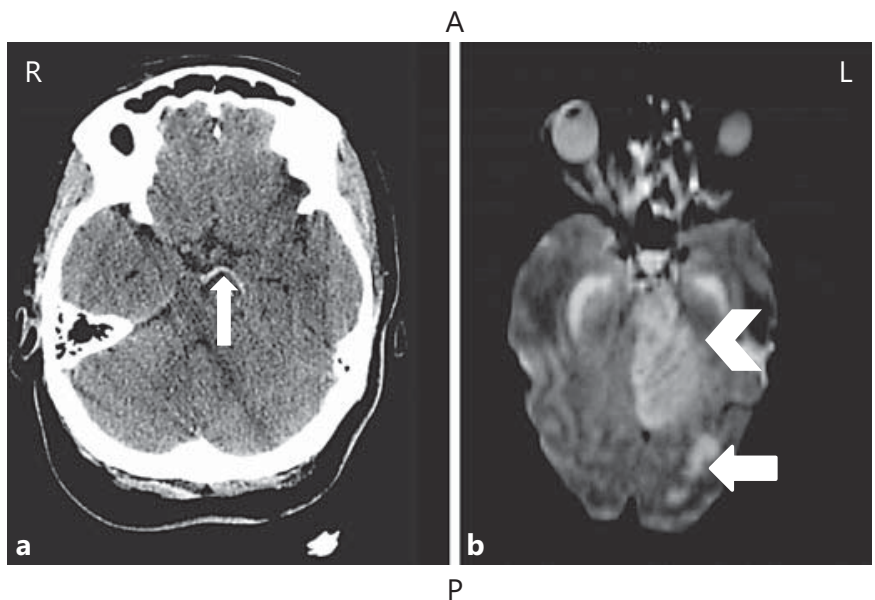

Fig. 1. The hyperdense PCA sign. $A=$ Anterior; $P=$ posterior; $R=$ right; $\mathrm{L}=$ left. a Noncontrast CT scan showing the hyperdense PCA sign (arrow) extending up to the basilar artery, suggestive of acute occlusion of the left PCA and basilar artery. $\mathbf{b}$ Magnetic resonance imaging (diffuse weighted image) showing an area of restricted diffusion, suggestive of acute infarction in the left medial temporal lobe (arrowhead) and occipital lobe (arrow), both supplied by the PCA.

ported cases the majority of the hyperdense PCA sign were attributed to embolic disease, either cardioembolic or vessel-to-vessel embolism from proximal stenosis of vertebral arteries [4-6]. The hyperdense artery sign is an indicator of occlusion of the involved vessel and precedes the radiological changes of acute ischemia seen on a noncontrast CT scan. Further, presence of the hyperdense artery sign is associated with larger areas of infarction and an unfavorable outcome, both in MCA as well as PCA territory strokes $[3,4]$. The 'hyperdense PCA sign' which our patient presented with which led to an urgent arteriogram enabled the timely diagnosis of vertebral dissection and intra-arterial thrombolysis, thus proving it to be a 'blessing in disguise' for this young female. Furthermore the basilar artery thrombosis that the patient had was the probable explanation for her obtunded state. Thus, the hyperdense PCA sign could point towards significant occlusive disease of the posterior circulation, such as basilar artery thrombosis which has significant mortality and morbidity. Prompt identification of basilar artery occlusion is critical for timely revascularization therapy (within $6 \mathrm{~h}$ of symptom onset) and to improve outcome [7]. Available meta-analysis data suggests no significant difference between two modalities of revascularization: intra-arterial thrombolysis and intravenous thrombolysis; however, early recanalization by either modality does improve functional outcome in a given subject [8]. There is 
lack of class I evidence to support any specific treatment modality such as antiplatelet versus anticoagulant as well as optimal duration of therapy in cases of vertebral artery dissection [9]. However, a large clot burden with a devastating clinical profile, like in our case, would necessitate use of recanalization and immediate anticoagulation therapy $[9,10]$.

\section{Conclusion}

This case showed an unusual occurrence of spontaneous vertebral dissection leading to embolic occlusion of the left PCA, demonstrated as hyperdense PCA sign on a $\mathrm{CT}$ scan. Hence we suggest an early intervention that may allow for potential revascularization therapy.

\section{References}

1 Koo CK, Teasdale E, Muir KW: What constitutes a true hyperdense middle cerebral artery sign? Cerebrovasc Dis 2000;10:419-423.

$>2$ Bakshi R, Mazziotta JC: Acute middle cerebral artery thrombosis demonstrated by cranial computed tomography: the 'dense MCA' sign. Arch Neurol 1998;55:1577.

$>3$ Schuknecht B, Ratzka M, Hofmann E: The 'dense artery sign' - major cerebral artery thromboembolism demonstrated by computed tomography. Neuroradiology 1990;32:98103.
4 Krings T, Noelchen D, Mull M, et al: The hyperdense posterior cerebral artery sign: a computed tomography marker of acute ischemia in the posterior cerebral artery territory. Stroke 2006;37:399-403.

5 Bettle N, Lyden PD: Thrombosis of the posterior cerebral artery (PCA) visualized on computed tomography: the dense PCA sign. Arch Neurol 2004;61:1960-1961.

$\checkmark 6$ Finelli PF: Neuroimaging in acute posterior cerebral artery infarction. Neurologist 2008; 14:170-180.

$>7$ Vergouwen MD, Algra A, Pfefferkorn T, et al, Basilar Artery International Cooperation Study (BASICS) Study Group: time is brain(stem) in basilar artery occlusion. Stroke 2012;43:3003-3006.
8 Mortimer AM, Bradley M, Renowden SA: Endovascular therapy for acute basilar artery occlusion: a review of the literature. J Neurointerv Surg 2012;4:266-273.

9 Mohan IV: Current optimal assessment and management of carotid and vertebral spontaneous and traumatic dissection. Angiology DOI: $10.1177 / 0003319712475154$.

10 Caplan LR: Dissections of brain-supplying arteries. Nat Clin Pract Neurol 2008;4:34. 